ESAIM: M2AN 47 (2013) 1845-1864

DOI: $10.1051 / \mathrm{m} 2 \mathrm{an} / 2013091$
ESAIM: Mathematical Modelling and Numerical Analysis

www.esaim-m2an.org

\title{
LOCAL DISCONTINUOUS GALERKIN METHODS FOR FRACTIONAL DIFFUSION EQUATIONS *,**
}

\author{
W.H. Deng ${ }^{1}$ AND J.S. Hesthaven ${ }^{2}$
}

\begin{abstract}
We consider the development and analysis of local discontinuous Galerkin methods for fractional diffusion problems in one space dimension, characterized by having fractional derivatives, parameterized by $\beta \in[1,2]$. After demonstrating that a classic approach fails to deliver optimal order of convergence, we introduce a modified local numerical flux which exhibits optimal order of convergence $\mathcal{O}\left(h^{k+1}\right)$ uniformly across the continuous range between pure advection $(\beta=1)$ and pure diffusion $(\beta=2)$. In the two classic limits, known schemes are recovered. We discuss stability and present an error analysis for the space semi-discretized scheme, which is supported through a few examples.
\end{abstract}

Mathematics Subject Classification. 35R11, 65M60, 65M12.

Received August 13, 2012. Revised May 21, 2013.

Published online October 7, 2013.

\section{INTRODUCTION}

The basic ideas behind fractional calculus have a history during three hundred year that is similar to and aligned with that of more classic calculus and the topic has attracted the interests of mathematicians who contributed fundamentally to the development of classical calculus, including L'Hospital, Leibniz, Liouville, Riemann, Grünward, and Letnikov [4]. In spite of this, the development and analysis of fractional calculus and fractional differential equations are not as mature as that associated with classical calculus. However, during the last decade this has begun to change as it has become clear that fractional calculus naturally emerges as a model for a broad range of non-classical phenomena in the applied sciences and engineering. A striking example of this is as a model for anomalous transport processes and diffusion, leading to partial differential equations (PDEs) of fractional type $[2,22]$. These models are found in a wide range of applications such as porous flows, models of a variety of biological processes, and transport in fusion plasmas, to name a few.

With this emerging range of applications and models based on fractional calculus comes a need for the development of robust and accurate computational methods for solving these equations. A fundamental difference

Keywords and phrases. Fractional derivatives, local discontinuous Galerkin methods, stability, convergence, error estimates.

* WHD was supported by CSC and in part by NNSFC Grant 10801067 and 11271173, NCET Grant NCET-09-0438, and the

Fundamental Research Funds for the Central Universities Grant lzujbky-2010-63.

** JSH was partially supported the National Science Foundation DMS-1115416.

1 School of Mathematics and Statistics, Lanzhou University, Lanzhou 730000, People's Republic of China; Division of Applied Mathematics, Brown University, 182 George Street, Providence, RI 02912, USA. dengwh@lzu.edu.cn

2 Division of Applied Mathematics, Brown University, 182 George Street, Providence, RI 02912, USA. Jan. Hesthaven@Brown.edu 
between problems in classic calculus and fractional calculus is the global nature of the latter formulations. Nevertheless, methods based on finite difference methods [23] and finite element formulations [10,11] have been developed and successfully applied.

The global nature of such models often leads to computational techniques which are substantially more resource intense than those associated with more classic problems. On the other hand, the solutions of fractional diffusion problem are generally endowed with substantial smoothness when the boundary conditions and/or the source term are specified properly. While these observations both suggest that it may be worth considering higher order accurate formulation as attractive alternatives, there appears to be very limited work in that direction with a few notable exceptions being $[5,16,17]$ in which spectral methods are used to discretize classical space derivative to solve time fractional PDEs and confirms the possible advantages of doing so.

In this work we introduce discontinuous Galerkin (DG) methods for the fractional diffusion problem in one space dimension. This development is built on the extensive work on DG for problems founded in classic calculus $[6,7,12,13,24]$. In particular we consider the extension of the local discontinuous Galerkin (LDG) method [6], based on previous work in [3], to problems containing fractional spatial derivatives. We find that the majority of the characteristics [13] of DG methods when used to solve classical PDEs carry over to fractional PDEs, i.e., the methods are naturally formulated for any order of accuracy in each element, there is flexibility in choosing element sizes in different places, allowing for adaptivity, and the mass matrix is local and easily invertible, leading to an explicit formulation for time dependent problems. We shall also discuss, however, that the choice of the numerical flux is essential to ensure the accuracy and stability of the scheme.

There is recent, yet limited, work along similar lines, e.g., in [18-21], where discontinuous Galerkin methods are discussed, although with an emphasis on first order methods and with a specific class of fractional problems in mind, primarily with the non classic operator being in time. Very recently [14] presents a purely qualitative study of the solution of spatial fractional problems in one and two dimensions using a high-order discontinuous Galerkin methods. However, no analysis or theoretical results are offered in this work.

We shall concern ourselves with equations on the form

$$
\frac{\partial u(x, t)}{\partial t}=d \frac{\partial^{\beta} u(x, t)}{\partial x^{\beta}}+f(x, t),
$$

subject to appropriate boundary conditions. Here $f(x, t)$ is a source term, $\beta \in[1,2]$, and $d \in R^{+}$, becoming a velocity in the advective limit $(\beta=1)$ and a pure diffusion coefficient in the pure diffusion limit of $(\beta=2)$. In the continuous range of $\beta \in(1,2)$ we refer to this as the generalized diffusion coefficient. In this non-classical range, we shall by $\frac{\partial^{\beta} u}{\partial x^{\beta}}$ understand the Riemann-Liouville derivative of order $\beta$. When $\beta=1$ and 2 , the Riemann-Liouville operator still makes sense and recovers exactly the first order and second order classical derivatives, respectively. Hence, within this framework we can unify the classical and fractional calculus and recover known formulations in the classic special cases.

To illustrate the importance of considering the development and analysis of discontinuous Galerkin methods for fractional problems, let us briefly consider the example

$$
\frac{\partial u(x, t)}{\partial t}=\frac{\Gamma(6-\beta)}{\Gamma(6)} \frac{\partial^{\beta} u(x, t)}{\partial x^{\beta}}-\mathrm{e}^{-t}\left(x^{5}+x^{5-\beta}\right)
$$

on the computational domain $x \in \Omega=(0,1)$. Here $\Gamma(x)$ is the classic Gamma function. We consider the initial condition $u(x, 0)=x^{5}$, subject to the Dirichlet boundary conditions $u(0, t)=0, u(1, t)=\mathrm{e}^{-t}$ and $\beta \in[1,2]$. The exact solution is given by $\mathrm{e}^{-t} x^{5}$. Following the classic work on local discontinuous Galerkin methods $[6,24]$, we express the fractional problem through new variables defined as

$$
\frac{\partial u(x, t)}{\partial t}-\sqrt{d} \frac{\partial q(x, t)}{\partial x}=f(x, t), q-{ }_{a} D_{x}^{\beta-2} p(x, t)=0, \quad p-\sqrt{d} \frac{\partial u(x, t)}{\partial x}=0,
$$

and discretize the two classic differential problems using a standard discontinuous Galerkin formulation with an alternating flux while recovery of $q$ from $p$ is obtained by a direct integration. Using this setup with a first 
TABLE 1. Error and order of convergence of a local discontinuous Galerkin method with a first order polynomial approximation $(k=1)$ when $\beta$ is very close to $1 . M$ denotes the number of elements.

\begin{tabular}{|c|c|c|c|c|l|l|l|l|l|}
\hline \multirow{2}{*}{$\beta$} & $M=2^{6}$ & \multicolumn{2}{|c|}{$M=2^{7}$} & \multicolumn{2}{c|}{$M=2^{8}$} & \multicolumn{2}{c|}{$M=2^{9}$} & \multicolumn{2}{c|}{$M=2^{10}$} \\
\cline { 2 - 10 } & error & error & order & error & order & error & order & error & order \\
\hline 1.10 & $5.66 \mathrm{e}-04$ & $1.90 \mathrm{e}-04$ & 1.58 & $5.22 \mathrm{e}-05$ & 1.86 & $1.32 \mathrm{e}-05$ & 1.98 & $3.31 \mathrm{e}-06$ & 2.00 \\
1.09 & $5.98 \mathrm{e}-04$ & $2.09 \mathrm{e}-04$ & 1.51 & $5.96 \mathrm{e}-05$ & 1.81 & $1.53 \mathrm{e}-05$ & 1.96 & $3.86 \mathrm{e}-06$ & 1.99 \\
1.08 & $6.32 \mathrm{e}-04$ & $2.31 \mathrm{e}-04$ & 1.45 & $6.85 \mathrm{e}-05$ & 1.76 & $1.78 \mathrm{e}-05$ & 1.94 & $4.53 \mathrm{e}-06$ & 1.98 \\
1.07 & $6.66 \mathrm{e}-04$ & $2.56 \mathrm{e}-04$ & 1.38 & $7.94 \mathrm{e}-05$ & 1.69 & $2.12 \mathrm{e}-05$ & 1.90 & $5.40 \mathrm{e}-06$ & 1.98 \\
1.06 & $7.00 \mathrm{e}-04$ & $2.82 \mathrm{e}-04$ & 1.31 & $9.26 \mathrm{e}-05$ & 1.61 & $2.57 \mathrm{e}-05$ & 1.85 & $6.52 \mathrm{e}-06$ & 1.98 \\
1.05 & $7.35 \mathrm{e}-04$ & $3.11 \mathrm{e}-04$ & 1.24 & $1.09 \mathrm{e}-04$ & 1.52 & $3.17 \mathrm{e}-05$ & 1.78 & $8.11 \mathrm{e}-06$ & 1.97 \\
1.04 & $7.69 \mathrm{e}-04$ & $3.43 \mathrm{e}-04$ & 1.17 & $1.29 \mathrm{e}-04$ & 1.41 & $4.01 \mathrm{e}-05$ & 1.68 & $1.04 \mathrm{e}-05$ & 1.95 \\
1.03 & $8.03 \mathrm{e}-04$ & $3.75 \mathrm{e}-04$ & 1.10 & $1.52 \mathrm{e}-04$ & 1.30 & $5.18 \mathrm{e}-05$ & 1.55 & $1.45 \mathrm{e}-05$ & 1.84 \\
1.02 & $8.36 \mathrm{e}-04$ & $4.10 \mathrm{e}-04$ & 1.03 & $1.80 \mathrm{e}-04$ & 1.19 & $6.89 \mathrm{e}-05$ & 1.39 & $2.16 \mathrm{e}-05$ & 1.67 \\
1.01 & $8.69 \mathrm{e}-04$ & $4.46 \mathrm{e}-04$ & 0.96 & $2.12 \mathrm{e}-04$ & 1.07 & $9.31 \mathrm{e}-05$ & 1.19 & $3.44 \mathrm{e}-05$ & 1.44 \\
1.00 & $9.00 \mathrm{e}-04$ & $4.83 \mathrm{e}-04$ & 0.90 & $2.49 \mathrm{e}-04$ & 0.95 & $1.27 \mathrm{e}-04$ & 0.97 & $6.38 \mathrm{e}-05$ & 0.99 \\
\hline
\end{tabular}

order local approximations over $M$ elements, we show in Table 1 the accuracy and order of approximation for $\beta$ decreasing from 1.1 to 1.0. It is easily observed that there is a clear dependance of the rate of convergence on $\beta$, decreasing from second order to first order as $\beta$ approaches the classic limit of one. Results for other order show a similar reduction for the optimal $k+1$ to $k$ if the standard local discontinuous Galerkin and alternating flux is used. One of the main results of this work is to introduce a penalization that overcomes this reduction.

What remains of this paper is organized as follows. In Section 2, we review the definition and properties of fractional derivatives and introduce the appropriate functional setting. This sets the stage for Section 3 in which we introduce ways to specify appropriate initial and boundary conditions, and propose a new penalized local discontinuous Galerkin scheme and Section 4 contains the detailed stability and error analyses of the space semi-discretized schemes. In Section 5 the analysis is confirmed through extensive numerical results and Section 6 contains a few concluding remarks and outlook for future work.

\section{Preliminaries on fractional CAlCulus}

Below we offer the formal definitions of fractional derivatives and associated functional setting, required to proceed with the analysis in the subsequent sections.

\subsection{Fractional calculus}

The formal definition of the fractional integral emerges as a natural generalization of multiple integration. It is well-known that integrating a function $v(x) n$ times amounts to

$$
\int_{a}^{x} \mathrm{~d} \xi_{n} \int_{a}^{\xi_{n}} \mathrm{~d} \xi_{n-1} \ldots \int_{a}^{\xi_{2}} v\left(\xi_{1}\right) \mathrm{d} \xi_{1}=\frac{1}{(n-1) !} \int_{a}^{x}(x-\xi)^{n-1} v(\xi) \mathrm{d} \xi, \quad x>a,
$$

for which we introduce the notation

$$
{ }_{a} D_{x}^{-n} v(x)=\frac{1}{\Gamma(n)} \int_{a}^{x}(x-\xi)^{n-1} v(\xi) \mathrm{d} \xi, \quad x>a .
$$

Clearly, (2.1) still makes sense if we replace $n$ by $\alpha\left(\in \mathbb{R}^{+}\right)$, leading to the definition of the fractional integral,

$$
{ }_{a} D_{x}^{-\alpha} v(x)=\frac{1}{\Gamma(\alpha)} \int_{a}^{x}(x-\xi)^{\alpha-1} v(\xi) \mathrm{d} \xi, \quad x>a, \quad \alpha \in \mathbb{R}^{+},
$$

where $a \in \mathbb{R}$ and $a$ can be $-\infty$. 
Based on this, it could appear that the related definition of the fractional derivative can be obtained by simply mixing fractional integration with classical derivatives [4]. However, one quickly observes that there are several options for this definition. Indeed, there are at least three slightly different such definitions of fractional derivatives. In this work we focus on the two closely related Riemann-Liouville derivative and the Caputo derivative, respectively. The third form, known as the Grünwald-Letnikov derivative and based on ideas derived from finite difference methods, is equivalent to the Riemann-Liouville derivative provided the functions are sufficiently smooth.

The Riemann-Liouville derivative is recovered by first performing integration followed by classic differentiation, leading to the definition

$$
{ }_{a} D_{x}^{\alpha} v(x)=\frac{1}{\Gamma(n-\alpha)} \frac{d^{n}}{\mathrm{~d} x^{n}} \int_{a}^{x}(x-\xi)^{n-\alpha-1} v(\xi) \mathrm{d} \xi, \quad x>a, \quad \alpha \in[n-1, n) .
$$

Reversing the order of integration and differentiation results in the Caputo derivative of the form

$$
{ }_{a}^{C} D_{x}^{\alpha} v(x)=\frac{1}{\Gamma(n-\alpha)} \int_{a}^{x}(x-\xi)^{n-\alpha-1} \frac{d^{n} v(\xi)}{\mathrm{d} \xi^{n}} \mathrm{~d} \xi, \quad x>a, \quad \alpha \in[n-1, n) .
$$

From an analysis point of view, the Riemann-Liouville derivative is natural since we can define the operator ${ }_{a} D_{x}^{\alpha}(\alpha \in R)$ which 'connects' $-\infty$ to $+\infty$. When $\alpha$ are negative integers, zero, and positive integers, they correspond exactly to multiple integrations, the identity operator, and the classical derivatives [9], respectively.

More precisely, for $\alpha \in R^{+}$and $n$ a natural number, if we denote $\lim _{\alpha \rightarrow n^{+}}$as the right limit and $\lim _{\alpha \rightarrow n^{-}}$the left limit, respectively, we have

$$
\begin{gathered}
\lim _{\alpha \rightarrow 0^{+}}{ }_{a} D_{x}^{-\alpha} v(x)=\lim _{\alpha \rightarrow 0^{+}}{ }_{a} D_{x}^{\alpha} v(x)=v(x), \\
\lim _{\alpha \rightarrow(n-1)^{+}}{ }_{a} D_{x}^{-\alpha} v(x)={ }_{a} D_{x}^{-(n-1)} v(x), \quad \lim _{\alpha \rightarrow n^{-}}{ }_{a} D_{x}^{-\alpha} v(x)={ }_{a} D_{x}^{-n} v(x), \\
\lim _{\alpha \rightarrow(n-1)^{+}}{ }_{a} D_{x}^{\alpha} v(x)=\frac{d^{n-1} v(x)}{\mathrm{d} x^{n-1}}, \quad \lim _{\alpha \rightarrow n^{-}}{ }_{a} D_{x}^{\alpha} v(x)=\frac{d^{n} v(x)}{\mathrm{d} x^{n}}, \\
\lim _{\alpha \rightarrow(n-1)^{+}}{ }_{a}^{C} D_{x}^{\alpha} v(x)=\frac{d^{n-1} v(x)}{\mathrm{d} x^{n-1}}-\left.\frac{d^{n-1} v(x)}{\mathrm{d} x^{n-1}}\right|_{x=a}, \quad \lim _{\alpha \rightarrow n^{-}}{ }_{a}^{C} D_{x}^{\alpha} v(x)=\frac{d^{n} v(x)}{\mathrm{d} x^{n}} .
\end{gathered}
$$

The goal in this work is to develop computational methods which mirror these attractive properties to the extend possible.

The main advantage of considering the Caputo derivative is the ease by which we can specify the proper initial and boundary conditions of the fractional differential equations. Using (2.4), we need to provide the values of $\frac{d^{k} v(x)}{\mathrm{d} x^{k}}, k=0,1, \ldots, n-1$ at the boundaries, similar to the situation for the classical differential equations. In contrast to this, for the Riemann-Liouville derivative (2.3), one needs to specify values of ${ }_{a} D_{x}^{k+\alpha-n} v(x)$, $k=0,1, \ldots, n-1$ at the boundaries. While not a fundamental obstacle, it appears as a practical problem when solving problems for which the simple and geometric interpretation of the classic derivatives are more natural.

In this work, we exploit the advantages of both of the two forms of fractional derivatives and focus on problems where (1.1) is equivalent under the two forms. Situations for which is true are understood through [15],

$$
{ }_{a} D_{x}^{\alpha} v(x)={ }_{a}^{C} D_{x}^{\alpha} v(x) \text { if } v^{(k)}(a)=0, \quad k=0,1, \ldots, n-1,
$$

where $n$ is the smallest integer greater than or equal to $\alpha$, and $v(x)$ is sufficiently smooth. 
At times, (2.2) and (2.3) are referred to as the left Riemann-Liouville fractional integral and the left Riemann-Liouville fractional derivative, respectively. In some cases it is natural to consider the interval $[x, b]$ instead of $[a, x]$, leading to the right Riemann-Liouville fractional integral being defined as

$$
{ }_{x} D_{b}^{-\alpha} v(x)=\frac{1}{\Gamma(\alpha)} \int_{x}^{b}(\xi-x)^{\alpha-1} v(\xi) \mathrm{d} \xi, \quad x<b, \quad \alpha \in \mathbb{R}^{+},
$$

where $b \in \mathbb{R}$ and $b$ can be $+\infty$. For the two operators (2.2) and (2.10), we have the following result

Lemma 2.1 ([11]). The left and the right Riemann-Liouville fractional integral operators are adjoint w.r.t. the $L^{2}(a, b)$ inner product, i.e., for all $\alpha>0$,

$$
\left({ }_{a} D_{x}^{-\alpha} u, v\right)_{L^{2}(a, b)}=\left(u,{ }_{x} D_{b}^{-\alpha} v\right)_{L^{2}(a, b)} \quad \forall \alpha>0, a<b, \text { and } u, v \in L^{2}(a, b) .
$$

\subsection{Negative fractional norms and fractional integral spaces}

Recall the Fourier transform $\hat{v}(\omega)$ of $v(x)$ defined as

$$
\mathcal{F}(v(x))=\int_{-\infty}^{+\infty} \mathrm{e}^{-i x \cdot \omega} v(x) \mathrm{d} x=\hat{v}(\omega) .
$$

As in classic Fourier analysis, we define a higher norm for functions in $\widetilde{H}^{-\alpha}(\mathbb{R})$ in terms of the Fourier transform. We use the notation $\widetilde{H}$ instead of $H$ since the usual definition of the Sobolev space of order $-\alpha$ uses a factor $\left(1+|\omega|^{2}\right)^{-\alpha / 2}$ not $|\omega|^{-\alpha}$. In this subsection, $\alpha, \alpha_{1}$, and $\alpha_{2}$ belong to $(0,1)$.

Definition 2.2. Let $\alpha \in(0,1)$. Define the norm

$$
\|v\|_{\widetilde{H}^{-\alpha}(\mathbb{R})}:=\left\||\omega|^{-\alpha} \hat{v}\right\|_{L^{2}(\mathbb{R})},
$$

and let $\widetilde{H}^{-\alpha}(\mathbb{R})$ denote the closure of $C_{0}^{\infty}(\mathbb{R})$ w.r.t. $\|\cdot\|_{\widetilde{H}^{-\alpha}(\mathbb{R})}$.

In a similar fashion, we define the norm associated with the left Riemann-Liouville derivative

Definition 2.3. Let $\alpha \in(0,1)$. Define the norm

$$
\|v\|_{J_{L}^{-\alpha}(\mathbb{R})}:=\left\|{ }_{-\infty} D_{x}^{-\alpha} v\right\|_{L^{2}(\mathbb{R})},
$$

and let $J_{L}^{-\alpha}(\mathbb{R})$ denote the closure of $C_{0}^{\infty}(\mathbb{R})$ w.r.t. $\|\cdot\|_{J_{L}^{-\alpha}(\mathbb{R})}$,

as well as the the norm associated with the left Riemann-Liouville derivative

Definition 2.4. Let $\alpha \in(0,1)$. Define the norm

$$
\|v\|_{J_{R}^{-\alpha}(\mathbb{R})}:=\left\|{ }_{x} D_{\infty}^{-\alpha} v\right\|_{L^{2}(\mathbb{R})},
$$

and let $J_{R}^{-\alpha}(\mathbb{R})$ denote the closure of $C_{0}^{\infty}(\mathbb{R})$ w.r.t. $\|\cdot\|_{J_{R}^{-\alpha}(\mathbb{R})}$.

The three norms are closely related as stated in the following result 
Theorem 2.5. The three spaces $\widetilde{H}^{-\alpha}, J_{L}^{-\alpha}$, and $J_{R}^{-\alpha}$ are equal with equivalent norms.

Proof. The Fourier transforms of the left and right Riemann-Liouville fractional integrals are, respectively,

$$
\mathcal{F}\left({ }_{-\infty} D_{x}^{-\alpha} v(x)\right)=(i \omega)^{-\alpha} \hat{v}(\omega) ; \text { and } \mathcal{F}\left({ }_{x} D_{\infty}^{-\alpha} v(x)\right)=(-i \omega)^{-\alpha} \hat{v}(\omega) .
$$

Since

$$
\left|(i \omega)^{-\alpha}\right|=\left|(-i \omega)^{-\alpha}\right|=|\omega|^{-\alpha} .
$$

Applying Plancherel's theorem, we have

$$
\sqrt{2 \pi}\|v\|_{\widetilde{H}^{-\alpha}(\mathbb{R})}=\left\||\omega|^{-\alpha} \hat{v}\right\|_{L^{2}(\mathbb{R})}=\sqrt{2 \pi}\|v\|_{J_{L}^{-\alpha}}=\sqrt{2 \pi}\|v\|_{J_{R}^{-\alpha}} .
$$

Using the same idea in the proof of Lemma 2.4 of [11], we obtain

\section{Lemma 2.6.}

$$
\left({ }_{-\infty} D_{x}^{-\alpha} v,{ }_{x} D_{\infty}^{-\alpha} v\right)=\cos (\alpha \pi)\left\|_{-\infty} D_{x}^{-\alpha} v\right\|_{L^{2}(\mathbb{R})}^{2}=\cos (\alpha \pi)\|v\|_{\widetilde{H}^{-\alpha}(\mathbb{R})}^{2}
$$

Let us now restrict attention to the case in which $\operatorname{supp}(v) \subset \Omega=(a, b)$. Then ${ }_{-\infty} D_{x}^{-\alpha} v={ }_{a} D_{x}^{-\alpha} v$, and ${ }_{x} D_{\infty}^{-\alpha} v={ }_{x} D_{b}^{-\alpha} v$. Straightforward extension of the definitions given above yields

Definition 2.7. Define the spaces $\widetilde{H}_{0}^{-\alpha}(\Omega), J_{L, 0}^{-\alpha}(\Omega)$, and $J_{R, 0}^{-\alpha}(\Omega)$ as the closures of $C_{0}^{\infty}(\Omega)$ under their respective norms.

The following theorem gives the relations among the fractional integral spaces with different $\alpha$.

Theorem 2.8. If $-\alpha_{2}<-\alpha_{1}<0$, then $J_{L, 0}^{-\alpha_{1}}(\Omega)\left(\widetilde{H}_{0}^{-\alpha_{1}}(\Omega)\right.$ or $\left.J_{R, 0}^{-\alpha_{1}}(\Omega)\right)$ is embedded into $J_{L, 0}^{-\alpha_{2}}(\Omega)\left(\widetilde{H}_{0}^{-\alpha_{2}}(\Omega)\right.$ or $J_{R, 0}^{-\alpha_{2}}(\Omega)$ ), and $L^{2}(\Omega)$ is embedded into both of them.

Proof. Using the semigroup properties of fractional integral operators [4], we have

$$
{ }_{a} D_{x}^{-\alpha_{2}} v={ }_{a} D_{x}^{-\left(\alpha_{2}-\alpha_{1}\right)}{ }_{a} D_{x}^{-\alpha_{1}} v .
$$

Using Young's inequality [1] and the definition of the fractional integral, we have

$$
\begin{aligned}
\|v\|_{J_{L, 0}^{-\alpha_{2}(\Omega)}} & =\left\|{ }_{a} D_{x}^{-\alpha_{2}} v\right\|_{L^{2}(\Omega)}=\left\|_{a} D_{x}^{-\left(\alpha_{2}-\alpha_{1}\right)}{ }_{a} D_{x}^{-\alpha_{1}} v\right\|_{L^{2}(\Omega)} \\
& =\frac{1}{\Gamma\left(\alpha_{2}-\alpha_{1}\right)}\left\|x^{\alpha_{2}-\alpha_{1}-1} *_{a} D_{x}^{-\alpha_{1}} v\right\|_{L^{2}(\Omega)} \\
& \leqslant \frac{1}{\Gamma\left(\alpha_{2}-\alpha_{1}\right)}\left\|x^{\alpha_{2}-\alpha_{1}-1}\right\|_{L^{1}(\Omega)} \cdot\left\|_{a} D_{x}^{-\alpha_{1}} v\right\|_{L^{2}(\Omega)} \\
& =\frac{|a|^{\alpha_{2}-\alpha_{1}}+|b|^{\alpha_{2}-\alpha_{1}}}{\Gamma\left(\alpha_{2}-\alpha_{1}+1\right)}\|v\|_{J_{L, 0}^{-\alpha_{1}}(\Omega)},
\end{aligned}
$$

where $*$ means the convolution product. Continuing in a similar fashion, we have

$$
\|v\|_{J_{L, 0}^{-\alpha_{1}}(\Omega)}=\left\|{ }_{a} D_{x}^{-\alpha_{1}} v\right\|_{L^{2}(\Omega)} \leqslant \frac{|a|^{\alpha_{1}}+|b|^{\alpha_{1}}}{\Gamma\left(\alpha_{1}+1\right)}\|v\|_{L^{2}(\Omega)}
$$

from which the result follows.

Note. The definitions of the three norms can be naturally extended to the case $\alpha \in[0,1)$. In this case, all equals the $L^{2}$-norm when $\alpha=0$. 


\section{The local Discontinuous Galerkin sChemes For the FraCtional Diffusion EQUATION}

The fractional derivative can be treated in two ways: the Riemann-Liouville fractional derivative of order $\beta,{ }_{a} D_{x}^{\beta} u(x)$ or, alternatively, one classical derivative acting on the Riemann-Liouville fractional derivative of order $\beta-1$. Assuming that $u(a, t)=0$ it follows from (2.9) that ${ }_{a} D_{x}^{\beta-1} u(x, t)={ }_{a}^{C} D_{x}^{\beta-1} u(x, t)$, and we can easily identify and specify the boundary conditions of (1.1). This allows us to more clearly describe the model discussed here

$$
\frac{\partial u(x, t)}{\partial t}=\frac{\partial}{\partial x} d_{a} D_{x}^{\beta-2} \frac{\partial}{\partial x} u(x, t)+f(x, t) \quad \text { in } \Omega_{T}=(a, b) \times(0, T),
$$

with the initial condition

$$
u(x, 0)=u_{0}(x) \quad \text { on } \Omega=(a, b)
$$

and the Dirichlet boundary conditions

$$
u(a, t)=0, \quad u(b, t)=g(t) \quad \text { on }(0, T),
$$

where $\beta \in[1,2], d>0$; and $(a, b)$ is bounded. Note that the homogeneous left boundary condition is necessary. In the special case of $\beta=1$, the equation degenerates to a pure convection problem and only the boundary condition at $x=b$ is required.

We note that the decomposition in (3.1) is not unique, i.e., one could express the fractional derivative in several different ways such as

$$
\frac{\partial^{2}}{\partial x^{2}}{ }_{a} D_{x}^{\beta-2}, \quad \frac{\partial}{\partial x}{ }_{a} D_{x}^{\beta-2} \frac{\partial}{\partial x}, \quad{ }_{a} D_{x}^{\beta-2} \frac{\partial^{2}}{\partial x^{2}},
$$

by combining the classic and Riemann-Liouville operators in different ways. The choice we propose here is not only related to the need to specify boundary conditions in a straightforward manner, but the order also impacts the properties of the numerical scheme.

\subsection{The weak formulation}

Following the standard approach for the development of local discontinuous Galerkin methods for problems with higher derivatives $[6,13,24]$, let us introduce the auxiliary variables $p$ and $q$, and rewrite $(3.1)-(3.3)$ as

$$
\begin{array}{ll}
\frac{\partial u(x, t)}{\partial t}-\sqrt{d} \frac{\partial q(x, t)}{\partial x}=f(x, t) & \text { in } \Omega_{T}, \\
q-{ }_{a} D_{x}^{\beta-2} p(x, t)=0 & \text { in } \Omega_{T}, \\
p-\sqrt{d} \frac{\partial u(x, t)}{\partial x}=0 & \text { in } \Omega_{T}, \\
u(x, 0)=u_{0}(x) & \text { on } \Omega, \\
u(a, t)=0, \quad u(b, t)=g(t) & \text { on }(0, T) .
\end{array}
$$

Using standard notation, given the nodes $a=x_{0}<x_{1}<\ldots<x_{M-1}<x_{M}=b$, we define the mesh $\mathcal{T}=\left\{I_{j}=\left(x_{j-1}, x_{j}\right), j=1, \ldots, M\right\}$ and set $h_{j}:=\left|I_{j}\right|=x_{j}-x_{j-1} ;$ and $h:=\max _{j=1}^{M} h_{j}$. Associated with the mesh $\mathcal{T}$, we define the broken Sobolev spaces

$$
L^{2}(\Omega, \mathcal{T}):=\left\{v: \Omega \rightarrow \mathbb{R}|v|_{I_{j}} \in L^{2}\left(I_{j}\right), j=1, \ldots, M\right\}
$$


and

$$
H^{1}(\Omega, \mathcal{T}):=\left\{v: \Omega \rightarrow \mathbb{R}|v|_{I_{j}} \in H^{1}\left(I_{j}\right), j=1, \ldots, M\right\} .
$$

For a function $v \in H^{1}(\Omega, \mathcal{T})$, we denote the one-sided limits at the nodes $\left\{x_{j}\right\}$ by

$$
v^{ \pm}\left(x_{j}\right)=v\left(x_{j}^{ \pm}\right):=\lim _{x \rightarrow x_{j}^{ \pm}} v(x) .
$$

We assume that the exact solution $\mathbf{w}=(u, p, q)$ of (3.4) belongs to

$$
H^{1}\left(0, T ; H^{1}(\Omega, \mathcal{T})\right) \times L^{2}\left(0, T ; L^{2}(\Omega, \mathcal{T})\right) \times L^{2}\left(0, T ; H^{1}(\Omega, \mathcal{T})\right)
$$

This assumption is reasonable since both $H^{1}(\Omega)$ and $L^{2}(\Omega)$ are embedded in the fractional integral spaces. We require that $\mathbf{w}$ satisfies

$$
\begin{aligned}
\left(\frac{\partial u(x, t)}{\partial t}, v\right)_{I_{j}}+\sqrt{d}\left(q(x, t), \frac{\partial v}{\partial x}\right)_{I_{j}}-\left.\sqrt{d} q(x, t) v\right|_{x_{j-1}^{+}} ^{x_{j}^{-}} & =(f, v)_{I_{j}}, \\
(q, w)_{I_{j}}-\left({ }_{a} D_{x}^{\beta-2} p(x, t), w\right)_{I_{j}} & =0, \\
(p, z)_{I_{j}}+\sqrt{d}\left(u(x, t), \frac{\partial z}{\partial x}\right)_{I_{j}}-\left.\sqrt{d} u(x, t) z\right|_{x_{j-1}^{+}} ^{x_{j}^{-}} & =0, \\
(u(\cdot, 0), v)_{I_{j}} & =\left(u_{0}(\cdot), v\right)_{I_{j}},
\end{aligned}
$$

for all test functions $w \in L^{2}(\Omega, \mathcal{T})$, and $v, z \in H^{1}(\Omega, \mathcal{T})$, and for $j=1, \ldots, M$. Here the time derivative is understood in the weak sense and $(u, v)_{I}=\int_{I} u(x) v(x) \mathrm{d} x$ is the standard inner product over the element.

\subsection{The numerical schemes}

Let us in the following propose numerical schemes for (3.1)-(3.3) based on (3.7)-(3.10). The global nature of the fractional derivative is reflected in (3.8) while (3.7) and (3.9) remain local as in a traditional discontinuous Galerkin formulations.

We now restrict the trial and test functions $v, w$, and $z$ to the finite dimensional subspaces $V \subset H^{1}(\Omega, \mathcal{T})$, and choose $V$ to be the space of discontinuous, piecewise polynomials

$$
V=\left\{v: \Omega \rightarrow \mathbb{R}|v|_{I_{j}} \in \mathcal{P}^{k}\left(I_{j}\right), j=1, \ldots, M\right\},
$$

where $\mathcal{P}^{k}\left(I_{j}\right)$ denotes the set of all polynomials of degree less than or equal $k(\geqslant 1)$ on $I_{j}$. Furthermore, we define $U, P$, and $Q$ as the approximations of $u, p$, and $q$, respectively, in the space $V$. We seek $(U, P, Q) \in$ $H^{1}(0, T ; V) \times L^{2}(0, T ; V) \times L^{2}(0, T ; V)$ such that for all $(v, w, z) \in H^{1}(0, T ; V) \times L^{2}(0, T ; V) \times L^{2}(0, T ; V)$, and for $j=1, \ldots, M$ the following holds:

$$
\begin{aligned}
\left(\frac{\partial U(x, t)}{\partial t}, v\right)_{I_{j}}+\sqrt{d}\left(Q(x, t), \frac{\partial v}{\partial x}\right)_{I_{j}}-\left.\sqrt{d} \hat{Q}(x, t) v\right|_{x_{j-1}^{+}} ^{x_{j}^{-}} & =(f, v)_{I_{j}}, \\
(Q, w)_{I_{j}}-\left({ }_{a} D_{x}^{\beta-2} P(x, t), w\right)_{I_{j}} & =0 \\
(P, z)_{I_{j}}+\sqrt{d}\left(U(x, t), \frac{\partial z}{\partial x}\right)_{I_{j}}-\left.\sqrt{d} \hat{U}(x, t) z\right|_{x_{j-1}^{+}} ^{x_{j}^{-}} & =0, \\
(U(\cdot, 0), v)_{I_{j}} & =\left(u_{0}(\cdot), v\right)_{I_{j}} .
\end{aligned}
$$


To complete the formulation of the numerical schemes, we need to define the numerical fluxes $\hat{Q}(x, t)$ and $\hat{U}(x, t)$. As with traditional local discontinuous Galerkin methods, this choice is the most delicate one as it determines not only locality but also consistency, stability, and order of convergence of the scheme. Seeking inspiration in the mixed formulation for the heat equation it is natural to consider the 'alternating principle' [24] in choosing the numerical fluxes for (3.11) and (3.13), that is, we choose

$$
\hat{Q}\left(x_{j}, t\right)=Q^{+}\left(x_{j}, t\right), \quad \hat{U}\left(x_{j}, t\right)=U^{-}\left(x_{j}, t\right)
$$

or

$$
\hat{Q}\left(x_{j}, t\right)=Q^{-}\left(x_{j}, t\right), \quad \hat{U}\left(x_{j}, t\right)=U^{+}\left(x_{j}, t\right) ;
$$

at all interior boundaries. At the external boundaries we use

$$
\hat{Q}(a, t)=Q^{+}(a, t)=Q^{-}(a, t), \quad \hat{Q}(b, t)=Q^{-}(b, t)=Q^{+}(b, t)
$$

and

$$
\hat{U}(a, t)=0, \quad \hat{U}(b, t)=g(t),
$$

reflecting a Dirichlet boundary.

This is a classic local discontinuous Galerkin method, leading to the results already shown in Table 1. As we shall discuss in more detail shortly, this scheme, (3.11)-(3.14) with fluxes (3.15)-(3.18), is stable for any $\beta \in[1,2]$; and has the optimal convergent order $k+1$ for $\beta \in(1,2]$ and suboptimal convergent order $k$ for $\beta=1$ as already demonstrated.

To address this loss of optimality, we observe that when $\beta=1$ the numerical dissipation term disappears. We therefore introduce local dissipation to enhance the stability of the scheme at $\beta=1$ by adding a penalty term in (3.12).

This suggests a new scheme with

$$
(Q, w)_{I_{j}}-\left({ }_{a} D_{x}^{\beta-2} P(x, t), w\right)_{I_{j}}+\left.L(h, \beta) \cdot(\hat{P}(x, t) w)\right|_{x_{j-1}^{+}} ^{x_{j}^{-}}=0
$$

where

$$
\begin{aligned}
& \hat{P}\left(x_{j}^{-}, t\right)=\left[P\left(x_{j}, t\right)\right]:=P\left(x_{j}^{+}, t\right)-P\left(x_{j}^{-}, t\right) \\
& \hat{P}\left(x_{j-1}^{+}, t\right)=\left[P\left(x_{j-1}, t\right)\right]:=P\left(x_{j-1}^{+}, t\right)-P\left(x_{j-1}^{-}, t\right) .
\end{aligned}
$$

Here $L(h, \beta)$ is a positive constant depending on $\beta$ and $h$, the local cell size. As we shall discuss, this term recovers the optimal $k+1$ order of convergence for any $\beta \in[1,2]$. If the value of $L(h, \beta)$ is at least of order $h$, the order of convergence is $k+1$ for $\beta \in(1,2]$ (see Thm. 4.3). In the computational examples we take $L(h, \beta)=h^{\beta}$ in agreement with the scaling of the global operator.

\section{Stability AND ERROR ESTimates}

In this section we will develop the main theoretical analysis. The general scheme is expressed as: Find $(U, P, Q) \in H^{1}(0, T ; V) \times L^{2}(0, T ; V) \times L^{2}(0, T ; V)$ such that for all $(v, w, z) \in H^{1}(0, T ; V) \times L^{2}(0, T ; V) \times$ $L^{2}(0, T ; V)$, the following holds

$$
B(U, P, Q ; v, w, z)=\mathcal{L}(v, w, z) .
$$


Here $(U(\cdot, 0), v(\cdot, 0))=\left(u_{0}(\cdot), v(\cdot, 0)\right)$ and the discrete bilinear form $B$ and $\tilde{B}$ are defined as

$$
\begin{aligned}
\tilde{B}(U, P, Q ; v, w, z):=\int_{0}^{T}\left(\frac{\partial U(\cdot, t)}{\partial t}, v(\cdot, t)\right) \mathrm{d} t-\int_{0}^{T}\left({ }_{a} D_{x}^{\beta-2} P(x, t), w(x, t)\right) \mathrm{d} t \\
\quad+\sqrt{d} \int_{0}^{T}\left[\left(Q(x, t), \frac{\partial v(x, t)}{\partial x}\right)+\left(U(x, t), \frac{\partial z(x, t)}{\partial x}\right)\right] \mathrm{d} t+\int_{0}^{T}[(Q(\cdot, t), w(\cdot, t))+(P(\cdot, t), z(\cdot, t))] \mathrm{d} t \\
\quad+\sqrt{d} \int_{0}^{T}\left[\left(Q^{+}(a, t) v^{+}(a, t)-Q^{-}(b, t) v^{-}(b, t)\right)+\sum_{j=1}^{M-1}\left(\hat{Q}\left(x_{j}, t\right)[v]\left(x_{j}, t\right)+\hat{U}\left(x_{j}, t\right)[z]\left(x_{j}, t\right)\right)\right] \mathrm{d} t
\end{aligned}
$$

and

$$
\begin{aligned}
B(U, P, Q ; v, w, z):= & \tilde{B}(U, P, Q ; v, w, z) \\
& -L(h, \beta) \int_{0}^{T} \sum_{j=1}^{M-1}[P]\left(x_{j}, t\right)[w]\left(x_{j}, t\right) \mathrm{d} t .
\end{aligned}
$$

The discrete linear form $\mathcal{L}$ is given by

$$
\mathcal{L}(v, w, z)=\int_{0}^{T}(f(\cdot, t), v(\cdot, t)) \mathrm{d} t+\sqrt{d} \int_{0}^{T} g(t) z(b, t) \mathrm{d} t .
$$

Since the scheme is consistent with (3.4), the exact solution $(u, p, q)$ of (3.4) in the space (3.6) satisfies

$$
B(u, p, q ; v, w, z)=\mathcal{L}(v, w, z)
$$

for all $(v, w, z) \in H^{1}(0, T ; V) \times L^{2}(0, T ; V) \times L^{2}(0, T ; V)$.

\subsection{Numerical stability}

Let $(\widetilde{U}, \widetilde{P}, \widetilde{Q}) \in H^{1}(0, T ; V) \times L^{2}(0, T ; V) \times L^{2}(0, T ; V)$ be the perturbed solution of $(U, P, Q) ;$ i.e., $(\widetilde{U}, \widetilde{P}, \widetilde{Q})$ and $(U, P, Q)$ satisfy $(4.1)$ with different initial condition. We denote $e_{U}:=\widetilde{U}-U, e_{P}:=\widetilde{P}-P$, and $e_{Q}:=\widetilde{Q}-Q$ as the errors. Stability of the two schemes is established in the following theorem.

Theorem 4.1 ( $L^{2}$ stability). Scheme (4.1) is $L^{2}$ stable, and for all $t \in[0, T]$ their solution satisfies

$$
\begin{aligned}
\left\|e_{U}(\cdot, t)\right\|_{L^{2}(\Omega)}^{2}= & \left\|e_{U}(\cdot, 0)\right\|_{L^{2}(\Omega)}^{2}-2 \cos ((\beta / 2-1) \pi) \int_{0}^{t}\left\|e_{P}(\cdot, t)\right\|_{H^{-\left(1-\frac{\beta}{2}\right)}(\Omega)}^{2} \mathrm{~d} t \\
& -2 \cdot L(h, \beta) \int_{0}^{t} \sum_{j=1}^{M-1}\left[e_{P}\right]^{2}\left(x_{j}, t\right) \mathrm{d} t .
\end{aligned}
$$

Note. If the unstabilized scheme is considered in the limit of $\beta=1$ then $\left\|e_{U}(\cdot, t)\right\|_{L^{2}(\Omega)}^{2}=\left\|e_{U}(\cdot, 0)\right\|_{L^{2}(\Omega)}^{2}$, indicating that the numerical dissipation disappears. 
Proof of Theorem 4.1. It suffices to prove the result for the case $t=T$. From (4.1) we recover the perturbation equation

$$
B\left(e_{U}, e_{P}, e_{Q} ; v, w, z\right)=0,
$$

for all $(v, w, z) \in H^{1}(0, T ; V) \times L^{2}(0, T ; V) \times L^{2}(0, T ; V)$. Taking $v=e_{U}, w=-e_{P}, z=e_{Q}$, and using Lemmas 2.1 and 2.6, we obtain,

$$
\begin{aligned}
0= & B\left(e_{U}, e_{P}, e_{Q} ; e_{U},-e_{P}, e_{Q}\right) \\
= & \frac{1}{2} \int_{0}^{T} \frac{\partial}{\partial t}\left\|e_{U}(\cdot, t)\right\|_{L^{2}(\Omega)}^{2} \mathrm{~d} t+\cos ((\beta / 2-1) \pi) \int_{0}^{T}\left\|e_{P}\right\|_{H^{-\left(1-\frac{\beta}{2}\right)}(\Omega)}^{2} \mathrm{~d} t \\
& +L(h, \beta) \int_{0}^{T} \sum_{j=1}^{M-1}\left[e_{P}\right]^{2}\left(x_{j}, t\right) \mathrm{d} t+\sqrt{d} \int_{0}^{T} \int_{a}^{b} \frac{\partial\left(e_{U} \cdot e_{Q}\right)}{\partial x} \mathrm{~d} x \mathrm{~d} t \\
& +\sqrt{d} \int_{0}^{T} \sum_{j=1}^{M-1} \hat{e}_{Q}\left(x_{j}, t\right)\left[e_{U}\right]\left(x_{j}, t\right) \mathrm{d} t+\sqrt{d} \int_{0}^{T} \sum_{j=1}^{M-1} \hat{e}_{U}\left(x_{j}, t\right)\left[e_{Q}\right]\left(x_{j}, t\right) \mathrm{d} t \\
& +\sqrt{d} \int_{0}^{T}\left(e_{Q}^{+}(a, t) e_{U}^{+}(a, t)-e_{Q}^{-}(b, t) e_{U}^{-}(b, t)\right) \mathrm{d} t .
\end{aligned}
$$

In (4.8),

$$
\begin{aligned}
\int_{0}^{T} \int_{a}^{b} \frac{\partial\left(e_{U} \cdot e_{Q}\right)}{\partial x} \mathrm{~d} x \mathrm{~d} t= & \int_{0}^{T}\left(-e_{Q}^{+}(a, t) e_{U}^{+}(a, t)+e_{Q}^{-}(b, t) e_{U}^{-}(b, t)\right) \mathrm{d} t \\
& -\int_{0}^{T} \sum_{j=1}^{M-1}\left[e_{U} \cdot e_{Q}\right]\left(x_{j}, t\right),
\end{aligned}
$$

and when $\hat{e}_{Q}=\mathrm{e}_{Q}^{+}$and $\hat{e}_{U}=\mathrm{e}_{U}^{-}\left(\right.$or $\hat{e}_{Q}=\mathrm{e}_{Q}^{-}$and $\left.\hat{e}_{U}=\mathrm{e}_{U}^{+}\right)$,

$$
\begin{aligned}
& \int_{0}^{T} \sum_{j=1}^{M-1} \hat{e}_{Q}\left(x_{j}, t\right)\left[e_{U}\right]\left(x_{j}, t\right)+\sum_{j=1}^{M-1} \hat{e}_{U}\left(x_{j}, t\right)\left[e_{Q}\right]\left(x_{j}, t\right) \mathrm{d} t \\
& =\int_{0}^{T} \sum_{j=1}^{M-1}\left[e_{U} \cdot e_{Q}\right]\left(x_{j}, t\right) \mathrm{d} t .
\end{aligned}
$$

Combining (4.8) and (4.9)-(4.10), the result follows.

\subsection{Error estimates}

For the error analysis, we define the projection operators $\mathrm{P}^{ \pm}, \mathcal{S}$, and $\mathcal{S}^{\prime}$ from $H^{1}(\Omega, \mathcal{T})$ to $V$. For intervals $I_{j}=\left(x_{j-1}, x_{j}\right), j=1,2, \ldots, M$, and any sufficiently regular function $u, \mathrm{P}^{ \pm}$are defined to satisfy the $k+1$ conditions:

$$
\begin{aligned}
& \left(\mathrm{P}^{ \pm} u-u, v\right)_{I_{j}}=0 \forall v \in \mathcal{P}^{k-1}\left(I_{j}\right), \text { if } k>0, \\
& \mathrm{P}^{-} u\left(x_{j}\right)=u^{-}\left(x_{j}\right) \mathrm{P}^{+} u\left(x_{j-1}\right)=u^{+}\left(x_{j-1}\right) .
\end{aligned}
$$


$\mathcal{S}$ and $\mathcal{S}^{\prime}$ are the standard $L^{2}$-projections, which are defined, respectively, as

$$
\begin{gathered}
(\mathcal{S} u-u, v)_{I_{j}}=0 \quad \forall v \in \mathcal{P}^{k}\left(I_{j}\right), \\
\left(\mathcal{S}^{\prime} u-u, v\right)_{I_{j}}=0 \quad \forall v \in \mathcal{P}^{k-1}\left(I_{j}\right), \text { if } k>0 .
\end{gathered}
$$

We are now ready to state our results and then prove them. These results are obtained under the assumption that the corresponding analytical solutions are sufficiently regular as functions of $x$.

Theorem 4.2 (Error estimate). The error for the scheme (3.11), (3.19), (3.13), and (3.14) with fluxes (3.15) (or (3.16)), (3.20), applied to the model (3.1)-(3.3) satisfies

$$
\sqrt{\int_{a}^{b}(u(x, t)-U(x, t))^{2} \mathrm{~d} x} \leqslant\left\{\begin{array}{lc}
\left(\sqrt{\frac{L(h, \beta)}{h}} c+c(\beta) \exp (T)\right) h^{k+1} & \text { for } 1<\beta \leqslant 2, \\
c h^{k} & \text { for } \beta=1,
\end{array}\right.
$$

where $c(\beta)$ and $c$ depend on $\frac{\partial^{k+1} u(x, t)}{\partial x^{k+1}}, \frac{\partial^{k+\beta-1} u(x, t)}{\partial x^{k+\beta-1}}, \frac{\partial^{k+\beta} u(x, t)}{\partial x^{k+\beta}}$, and time $t$.

Note. For the current proof, the error bound derived for $\beta \in(1,2]$ is not uniform; but it may be uniform for any $\beta \in\left(\beta_{0}, 2\right]$, where $\beta_{0}>1$.

Proof of Theorem 4.2. We begin by proving the result for the unstabilized scheme and then outline the extension needed for the stabilized result.

We denote

$$
e_{u}=u(x, t)-U(x, t), \quad e_{p}=p(x, t)-P(x, t), \quad e_{q}=q(x, t)-Q(x, t) .
$$

From (4.1) and (4.5), we recover the error equation

$$
\tilde{B}\left(e_{u}, e_{p}, e_{q} ; v, w, z\right)=0
$$

for all $(v, w, z) \in H^{1}(0, T ; V) \times L^{2}(0, T ; V) \times L^{2}(0, T ; V)$. Take

$$
v=\mathrm{P}^{ \pm} u-U, \quad w=P-\mathcal{S} p, \quad z=\mathrm{P}^{\mp} q-Q
$$

in (4.15). After rearranging terms, we obtain

$$
\tilde{B}(v,-w, z ; v, w, z)=\tilde{B}\left(v^{e},-w^{e}, z^{e} ; v, w, z\right),
$$

where $v^{e}, w^{e}$, and $z^{e}$ are given as

$$
v^{e}=\mathrm{P}^{ \pm} u-u, \quad w^{e}=p-\mathcal{S} p, \quad z^{e}=\mathrm{P}^{\mp} q-q .
$$

Following the discussion in the Proof of Theorem 4.1 the left hand side of (4.16) becomes

$$
\tilde{B}(v,-w, z ; v, w, z)=\frac{1}{2} \int_{0}^{T} \frac{\partial}{\partial t}\|v(\cdot, t)\|_{L^{2}(\Omega)}^{2} \mathrm{~d} t+\cos ((\beta / 2-1) \pi) \int_{0}^{T}\|w(\cdot, t)\|_{H^{-\left(1-\frac{\beta}{2}\right)}(\Omega)}^{2} \mathrm{~d} t .
$$


Using the notation in [24], the right hand side of (4.16) can be expressed as

$$
\tilde{B}\left(v^{e},-w^{e}, z^{e} ; v, w, z\right)=\mathcal{I}+\mathcal{I} \mathcal{I}+\mathcal{I} \mathcal{I}+\mathcal{I} \mathcal{V}+\mathcal{V}
$$

where

$$
\begin{gathered}
\mathcal{I}=\int_{0}^{T}\left(\frac{\partial v^{e}(\cdot, t)}{\partial t}, v(\cdot, t)\right) \mathrm{d} t, \\
\mathcal{I} \mathcal{I}=\sqrt{d} \int_{0}^{T}\left(z^{e}(x, t), \frac{\partial v(x, t)}{\partial x}\right) \mathrm{d} t+\sqrt{d} \int_{0}^{T}\left(v^{e}(x, t), \frac{\partial z(x, t)}{\partial x}\right) \mathrm{d} t-\int_{0}^{T}\left(w^{e}(\cdot, t), z(\cdot, t)\right) \mathrm{d} t, \\
\mathcal{I I I}=\sqrt{d} \int_{0}^{T} \sum_{j=1}^{M-1} \hat{z}^{e}\left(x_{j}, t\right)[v]\left(x_{j}, t\right) \mathrm{d} t+\sqrt{d} \int_{0}^{T} \sum_{j=1}^{M-1} \hat{v}^{e}\left(x_{j}, t\right)[z]\left(x_{j}, t\right) \mathrm{d} t, \\
\mathcal{I} \mathcal{V}=\sqrt{d} \int_{0}^{T}\left(\left(z^{e}\right)^{+}(a, t) v^{+}(a, t)-\left(z^{e}\right)^{-}(b, t) v^{-}(b, t)\right) \mathrm{d} t,
\end{gathered}
$$

and

$$
\mathcal{V}=\int_{0}^{T}\left(z^{e}(\cdot, t), w(\cdot, t)\right) \mathrm{d} t+\int_{0}^{T}\left(\frac{\partial^{\beta-2} w^{e}(x, t)}{\partial x^{\beta-2}}, w(x, t)\right) \mathrm{d} t
$$

Using standard approximation theory [8], we obtain

$$
\begin{aligned}
\mathcal{I} & \leqslant \frac{1}{2} \int_{0}^{T} \int_{a}^{b}\left(\frac{\partial v^{e}(x, t)}{\partial t}\right)^{2} \mathrm{~d} x \mathrm{~d} t+\int_{0}^{T} \int_{a}^{b}\left(\frac{v^{2}(x, t)}{2}\right) \mathrm{d} x \mathrm{~d} t \\
& \leqslant c h^{2 k+2}+\frac{1}{2} \int_{0}^{T}\|v(\cdot, t)\|_{L^{2}(\Omega)}^{2} \mathrm{~d} t
\end{aligned}
$$

where $c$ is constant.

All the terms in $\mathcal{I} \mathcal{I}$ vanish due to Galerkin orthogonality, i.e., $p-\mathcal{S} p$ is orthogonal to all polynomials of degree up to $k$, and $\mathrm{P}^{ \pm} u-u$ and $\mathrm{P}^{ \pm} q-q$ to $k-1$. For the terms in $\mathcal{I} \mathcal{I}$, when taking $\hat{z}^{e}=\left(z^{e}\right)^{-}$and $\hat{v}^{e}=\left(v^{e}\right)^{+}$, we use $z^{e}=\mathrm{P}^{-} q-q$ and $v^{e}=\mathrm{P}^{+} u-u$; and when giving $\hat{z}^{e}=\left(z^{e}\right)^{+}$and $\hat{v}^{e}=\left(v^{e}\right)^{-}$, we choose $z^{e}=\mathrm{P}^{+} q-q$ and $v^{e}=\mathrm{P}^{-} u-u$. Hence, both terms in $\mathcal{I} \mathcal{I}$ are zero and so is $\mathcal{I} \mathcal{I}$. An application of the inequality $x y \leqslant \frac{1}{2}\left(x^{2}+y^{2}\right)$, the standard approximation of point values of $z^{e}$, and the equivalence of norms in finite dimensional spaces imply

$$
\begin{aligned}
\mathcal{I} \mathcal{V} & \leqslant \frac{\sqrt{d}}{2} \int_{0}^{T}\left(\left(\left(z^{e}\right)^{+}(a, t)\right)^{2}+\left(\left(z^{e}\right)^{-}(b, t)\right)^{2}+\left(v^{+}(a, t)\right)^{2}+\left(v^{-}(b, t)\right)^{2}\right) \mathrm{d} t \\
& \leqslant c h^{2 k+2}+c \int_{0}^{T}\|v(\cdot, t)\|_{L^{\infty}(\Omega)}^{2} \mathrm{~d} t \leqslant c h^{2 k+2}+c \int_{0}^{T}\|v(\cdot, t)\|_{L^{2}(\Omega)}^{2} \mathrm{~d} t .
\end{aligned}
$$

One of the terms in $\mathcal{I} \mathcal{V}$ is exactly zero. When $z^{e}=\mathrm{P}^{+} q-q$, the first term is zero; and when $z^{e}=\mathrm{P}^{-} q-q$, the second term is zero. For $\mathcal{V}$, we recover two different kinds of estimates corresponding to $\beta=1$ and $\beta \in(1,2]$ respectively. Both of them use Lemma 2.1. 
When $\beta=1$, further applying the operators $\mathcal{S}$ and $\mathcal{S}^{\prime}$ defined in (4.12) and (4.13) yields

$$
\begin{aligned}
\mathcal{V}= & \int_{0}^{T}\left(z^{e}(\cdot, t), w(\cdot, t)-\mathcal{S}^{\prime} w(\cdot, t)\right)+\left(z^{e}(\cdot, t), \mathcal{S}^{\prime} w(\cdot, t)\right) \mathrm{d} t \\
& +\int_{0}^{T}\left(w^{e}(\cdot, t), \int_{x}^{b} w(\xi, t) \mathrm{d} \xi-\mathcal{S} \int_{x}^{b} w(\xi, t) \mathrm{d} \xi\right)+\left(w^{e}(\cdot, t), \mathcal{S} \int_{x}^{b} w(\xi, t) \mathrm{d} \xi\right) \mathrm{d} t \\
= & \int_{0}^{T}\left(z^{e}(\cdot, t), w(\cdot, t)-\mathcal{S}^{\prime} w(\cdot, t)\right)+\left(w^{e}(\cdot, t), \int_{x}^{b} w(\xi, t) \mathrm{d} \xi-\mathcal{S} \int_{x}^{b} w(\xi, t) \mathrm{d} \xi\right) \mathrm{d} t \\
\leqslant & \int_{0}^{T} \int_{a}^{b}\left(\frac{\left(z^{e}(x, t)\right)^{2}}{2}\right) \mathrm{d} x \mathrm{~d} t+\int_{0}^{T} \int_{a}^{b}\left(\frac{\left(w(x, t)-\mathcal{S}^{\prime} w(x, t)\right)^{2}}{2}\right) \mathrm{d} x \mathrm{~d} t \\
& +\frac{1}{2} \int_{0}^{T} \int_{a}^{b}\left(\frac{\left(w^{e}(x, t)\right)^{2}}{2}+\left(\int_{x}^{b} w(\xi, t) \mathrm{d} x-\mathcal{S} \int_{x}^{b} w(\xi, t) \mathrm{d} \xi\right)^{2}\right) \mathrm{d} x \mathrm{~d} t \\
\leqslant & c h^{2 k+2}+c h^{2 k}+c h^{2 k+2}+c h^{2 k+2} \leqslant c h^{2 k} .
\end{aligned}
$$

When $\beta \in(1,2]$, the inequality $x y \leqslant \frac{x^{2}}{2 \epsilon}+\frac{\epsilon y^{2}}{2}$ and the norm-equivalence gives

$$
\begin{aligned}
\mathcal{V} \leqslant & \int_{0}^{T} \int_{a}^{b} \frac{\left(z^{e}(x, t)\right)^{2}}{2 \epsilon} \mathrm{d} x \mathrm{~d} t+\frac{\epsilon}{2} \int_{0}^{T}\|w(\cdot, t)\|_{L^{2}(\Omega)}^{2} \mathrm{~d} t \\
& +\int_{0}^{T} \int_{a}^{b} \frac{\left(w^{e}(x, t)\right)^{2}}{2 \epsilon} \mathrm{d} x \mathrm{~d} t+\frac{\epsilon}{2} \int_{0}^{T}\|w(\cdot, t)\|_{H^{-(2-\beta)}(\Omega)}^{2} \mathrm{~d} t \\
\leqslant & (c / \epsilon) h^{2 k+2}+c \epsilon \int_{0}^{T}\|w(\cdot, t)\|_{H^{-\left(1-\frac{\beta}{2}\right)}(\Omega)}^{2} \mathrm{~d} t
\end{aligned}
$$

where $\epsilon$ is a small number, chosen such that the term in this equation can be controlled by the corresponding term in (4.17), namely, choosing a sufficiently small $\epsilon$ such that $c \epsilon<\cos ((\beta / 2-1) \pi)$; the fact that all norms in finite dimensional spaces are equivalent is needed, since $w(\cdot, t)$ is a polynomial. Combining the above estimates we have, for $\beta \in(1,2]$,

$$
\frac{1}{2} \int_{0}^{T} \frac{\partial}{\partial t}\|v(\cdot, t)\|_{L^{2}(\Omega)}^{2}+(\cos ((\beta / 2-1) \pi)-c \epsilon) \int_{0}^{T}\|w(\cdot, t)\|_{H^{-\left(1-\frac{\beta}{2}\right)}(\Omega)}^{2} \mathrm{~d} t \leqslant(c / \epsilon) h^{2 k+2}+c \int_{0}^{T}\|v(\cdot, t)\|_{L^{2}(\Omega)}^{2} \mathrm{~d} t
$$

which we express as

$$
\begin{aligned}
& \frac{1}{2}\|v(\cdot, T)\|_{L^{2}(\Omega)}^{2}+(\cos ((\beta / 2-1) \pi)-c \epsilon) \int_{0}^{T}\|w(\cdot, t)\|_{H^{-\left(1-\frac{\beta}{2}\right)}(\Omega)}^{2} \mathrm{~d} t \\
& \leqslant \frac{1}{2}\|v(\cdot, 0)\|_{L^{2}(\Omega)}^{2}+(c / \epsilon) h^{2 k+2}+c \int_{0}^{T}\|v(\cdot, t)\|_{L^{2}(\Omega)}^{2} \mathrm{~d} t \\
& \leqslant(c / \epsilon) h^{2 k+2}+c \int_{0}^{T}\|v(\cdot, t)\|_{L^{2}(\Omega)}^{2} \mathrm{~d} t .
\end{aligned}
$$


For the case of $\beta=1$,

$$
\frac{1}{2} \int_{0}^{T} \frac{\partial}{\partial t}\|v(\cdot, t)\|_{L^{2}(\Omega)}^{2} \leqslant c h^{2 k}+c \int_{0}^{T}\|v(\cdot, t)\|_{L^{2}(\Omega)}^{2} \mathrm{~d} t,
$$

such that

$$
\frac{1}{2}\|v(\cdot, T)\|_{L^{2}(\Omega)}^{2} \leqslant \frac{1}{2}\|v(\cdot, 0)\|_{L^{2}(\Omega)}^{2}+c h^{2 k}+c \int_{0}^{T}\|v(\cdot, t)\|_{L^{2}(\Omega)}^{2} \mathrm{~d} t \leqslant c h^{2 k}+c \int_{0}^{T}\|v(\cdot, t)\|_{L^{2}(\Omega)}^{2} \mathrm{~d} t .
$$

According to Grönwall's lemma [8] and the standard approximation on $v^{e}=\mathrm{P}^{ \pm} u-u$ the desired estimate is recovered for the unstabilized scheme.

Let us now briefly consider the impact of the stabilization term. The error equation is

$$
B\left(e_{u}, e_{p}, e_{q} ; v, w, z\right)=0,
$$

or

$$
\tilde{B}\left(e_{u}, e_{p}, e_{q} ; v, w, z\right)-L(h, \beta) \int_{0}^{T} \sum_{j=1}^{M-1}\left[e_{p}\right]\left(x_{j}, t\right)[w]\left(x_{j}, t\right) \mathrm{d} t=0 .
$$

After rearranging terms, we obtain

$$
B(v,-w, z ; v, w, z)=B\left(v^{e},-w^{e}, z^{e} ; v, w, z\right),
$$

or

$$
\tilde{B}(v,-w, z ; v, w, z)+\mathcal{V} \mathcal{I}^{\prime}=\tilde{B}\left(v^{e},-w^{e}, z^{e} ; v, w, z\right)+\mathcal{V} \mathcal{I}
$$

where

$$
\mathcal{V} \mathcal{I}^{\prime}=L(h, \beta) \int_{0}^{T} \sum_{j=1}^{M-1}[w]^{2}\left(x_{j}, t\right) \mathrm{d} t
$$

and

$$
\mathcal{V} \mathcal{I}=L(h, \beta) \int_{0}^{T} \sum_{j=1}^{M-1}\left[w^{e}\right]\left(x_{j}, t\right)[w]\left(x_{j}, t\right) \mathrm{d} t
$$

The estimate of $\mathcal{V I}$ is

$$
\mathcal{V} \mathcal{I} \leqslant L(h, \beta) \int_{0}^{T} \frac{1}{2} \sum_{j=1}^{M-1}\left(\left[w^{e}\right]^{2}\left(x_{j}, t\right)+[w]^{2}\left(x_{j}, t\right)\right) \mathrm{d} t \leqslant L(h, \beta)\left(c h^{2 k+1}+\frac{1}{2} \mathcal{V} \mathcal{I}^{\prime}\right) .
$$

We now have the final estimate, for $\beta \in(1,2]$,

$$
\begin{aligned}
\frac{1}{2}\|v(\cdot, T)\|_{L^{2}(\Omega)}^{2}+ & (\cos ((\beta / 2-1) \pi)-c \epsilon) \int_{0}^{T}\|w(\cdot, t)\|_{H^{-\left(1-\frac{\beta}{2}\right)}(\Omega)}^{2} \mathrm{~d} t+\frac{L(h, \beta)}{2} \int_{0}^{T} \sum_{j=1}^{M-1}[w]^{2}\left(x_{j}, t\right) \mathrm{d} t \\
& \leqslant L(h, \beta) c h^{2 k+1}+(c / \epsilon) h^{2 k+2}+c \int_{0}^{T}\|v(\cdot, t)\|_{L^{2}(\Omega)}^{2} \mathrm{~d} t \\
& \leqslant\left(\frac{L(h, \beta)}{h} c+(c / \epsilon)\right) h^{2 k+2}+c \int_{0}^{T}\|v(\cdot, t)\|_{L^{2}(\Omega)}^{2} \mathrm{~d} t .
\end{aligned}
$$


For the special case of $\beta=1$,

$$
\begin{aligned}
\frac{1}{2}\|v(\cdot, T)\|_{L^{2}(\Omega)}^{2}+ & \frac{L(h, \beta)}{2} \int_{0}^{T} \sum_{j=1}^{M-1}[w]^{2}\left(x_{j}, t\right) \mathrm{d} t \\
& \leqslant L(h, \beta) c h^{2 k+1}+c h^{2 k}+c \int_{0}^{T}\|v(\cdot, t)\|_{L^{2}(\Omega)}^{2} \mathrm{~d} t \leqslant c h^{2 k}+c \int_{0}^{T}\|v(\cdot, t)\|_{L^{2}(\Omega)}^{2} \mathrm{~d} t .
\end{aligned}
$$

Again using Grönwall's lemma recovers the desired estimate (4.14).

Note. Since the control term for $w$ in (4.17) disappears when $\beta=1$, an attempt to overcome this in the first term of $\mathcal{V}$ is to take $z^{e}=\mathcal{S} q-q$ instead of $z^{e}=\mathrm{P}^{\mp} q-q$, making this term vanish. However, in this case the first term of $\mathcal{I} \mathcal{I}$ no longer vanishes and we have

$$
\begin{aligned}
\mathcal{I} \mathcal{I} & \leqslant \sqrt{d} \int_{0}^{T} \sum_{j=1}^{M-1}\left(\frac{\left(\hat{z}^{e}\left(x_{j}, t\right)\right)^{2}}{2}+\frac{\left([v]\left(x_{j}, t\right)\right)^{2}}{2}\right) \mathrm{d} t \\
& \leqslant \int_{0}^{T} \sum_{j=1}^{M-1}\left(c h^{2 k+2}+c\|v(\cdot, t)\|_{L^{\infty}\left(I_{j}\right)}^{2}\right) \mathrm{d} t \leqslant c h^{2 k+1}+\int_{0}^{T} \frac{c}{h}\|v(\cdot, t)\|_{L^{2}(\Omega)}^{2} \mathrm{~d} t .
\end{aligned}
$$

The result in the final estimate is

$$
\frac{1}{2}\|v(\cdot, T)\|_{L^{2}(\Omega)}^{2} \leqslant h^{2 k+1}+\int_{0}^{T} \frac{c}{h}\|v(\cdot, t)\|_{L^{2}(\Omega)}^{2} \mathrm{~d} t .
$$

This estimate appears not to be useful. From the proof of the estimates for $\beta \in(1,2]$ we observe that if $\beta$ decreases then the constant $c / \epsilon$ in front of the order term increases. This is because $\cos ((\beta / 2-1) \pi)$ decreases when $\beta$ decreases, i.e., we can expect that $c(\beta)$ in the Theorem 4.2 increases as $\beta$ decreases towards one. The numerical results in the next section will confirm this.

The introduction of the terms $\mathcal{V} \mathcal{I}^{\prime}$ and $\mathcal{V} \mathcal{I}$ does not appear to add any additional freedom to improve on the error estimates $\left(\mathcal{V I} \mathcal{I}^{\prime}\right.$ can't control $\|w\|^{2}$, so we can not improve the order of convergence when $\left.\beta=1\right)$. On the contrary, we have to estimate the trace errors of $w$. It seems hard to choose a special projection to eliminate the traces errors, since at one point we have two traces of $w$. It is not clear whether the error estimate (4.14) is sharp. However, the numerical computations below show that the order of convergence is $k+1$ for any $\beta \in[1,1+\varepsilon]$, where $\varepsilon$ may be 0.01 or 0.001 and full convergence order is recovered for the entire interval with the penalization.

\section{Numerical ReSults}

Let us offer some numerical results to validate the analysis. We mainly focus on the accuracy and stability of the spatial approximation although we also numerically study the CFL condition associated with the fully discrete stability. We use a fourth order explicit Runge-Kutta method to solve the method-of-line fractional $\mathrm{PDE}$, i.e., the classical ODE system. To ensure the overall error is dominated by space error, small time steps are used.

We consider the problem

$$
\frac{\partial u(x, t)}{\partial t}=\frac{\Gamma(6-\beta)}{\Gamma(6)} \frac{\partial^{\beta} u(x, t)}{\partial x^{\beta}}-\mathrm{e}^{-t}\left(x^{5}+x^{5-\beta}\right),
$$


TABLE 2. The error and order of convergence for first order polynomial approximation $(k=1)$. $M$ denotes the number of elements. For $\beta>1.1$ the results are independent of whether stabilization with $L(h, \beta)=h^{\beta}$ is included. Recovery of the optimal order of convergence is possible only with the stabilization as illustrated in the last two rows which show results with the stabilized scheme.

\begin{tabular}{|l|c|c|l|l|l|l|l|l|l|}
\hline \multirow{2}{*}{$\beta$} & $M=2^{5}$ & \multicolumn{2}{|c|}{$M=2^{6}$} & \multicolumn{2}{c|}{$M=2^{7}$} & \multicolumn{2}{c|}{$M=2^{8}$} & \multicolumn{2}{c|}{$M=2^{9}$} \\
\cline { 2 - 9 } & error & error & order & error & order & error & order & error & order \\
\hline 2.00 & $2.81 \mathrm{e}-04$ & $7.82 \mathrm{e}-05$ & 1.85 & $2.04 \mathrm{e}-05$ & 1.94 & $5.20 \mathrm{e}-06$ & 1.97 & $1.31 \mathrm{e}-06$ & 1.99 \\
1.80 & $3.37 \mathrm{e}-04$ & $9.37 \mathrm{e}-05$ & 1.85 & $2.44 \mathrm{e}-05$ & 1.94 & $6.14 \mathrm{e}-06$ & 1.99 & $1.52 \mathrm{e}-06$ & 2.01 \\
1.50 & $4.63 \mathrm{e}-04$ & $1.27 \mathrm{e}-04$ & 1.87 & $3.23 \mathrm{e}-05$ & 1.98 & $7.93 \mathrm{e}-06$ & 2.03 & $1.95 \mathrm{e}-06$ & 2.02 \\
1.20 & $9.82 \mathrm{e}-04$ & $3.17 \mathrm{e}-04$ & 1.63 & $8.32 \mathrm{e}-05$ & 1.93 & $2.02 \mathrm{e}-05$ & 2.05 & $5.09 \mathrm{e}-06$ & 1.99 \\
1.10 & $5.66 \mathrm{e}-04$ & $1.90 \mathrm{e}-04$ & 1.58 & $5.22 \mathrm{e}-05$ & 1.86 & $1.32 \mathrm{e}-05$ & 1.98 & $3.31 \mathrm{e}-06$ & 2.00 \\
1.09 & $5.98 \mathrm{e}-04$ & $2.09 \mathrm{e}-04$ & 1.51 & $5.96 \mathrm{e}-05$ & 1.81 & $1.53 \mathrm{e}-05$ & 1.96 & $3.86 \mathrm{e}-06$ & 1.99 \\
1.08 & $6.32 \mathrm{e}-04$ & $2.31 \mathrm{e}-04$ & 1.45 & $6.85 \mathrm{e}-05$ & 1.76 & $1.78 \mathrm{e}-05$ & 1.94 & $4.53 \mathrm{e}-06$ & 1.98 \\
1.07 & $6.66 \mathrm{e}-04$ & $2.56 \mathrm{e}-04$ & 1.38 & $7.94 \mathrm{e}-05$ & 1.69 & $2.12 \mathrm{e}-05$ & 1.90 & $5.40 \mathrm{e}-06$ & 1.98 \\
1.06 & $7.00 \mathrm{e}-04$ & $2.82 \mathrm{e}-04$ & 1.31 & $9.26 \mathrm{e}-05$ & 1.61 & $2.57 \mathrm{e}-05$ & 1.85 & $6.52 \mathrm{e}-06$ & 1.98 \\
1.05 & $7.35 \mathrm{e}-04$ & $3.11 \mathrm{e}-04$ & 1.24 & $1.09 \mathrm{e}-04$ & 1.52 & $3.17 \mathrm{e}-05$ & 1.78 & $8.11 \mathrm{e}-06$ & 1.97 \\
1.04 & $7.69 \mathrm{e}-04$ & $3.43 \mathrm{e}-04$ & 1.17 & $1.29 \mathrm{e}-04$ & 1.41 & $4.01 \mathrm{e}-05$ & 1.68 & $1.04 \mathrm{e}-05$ & 1.95 \\
1.03 & $8.03 \mathrm{e}-04$ & $3.75 \mathrm{e}-04$ & 1.10 & $1.52 \mathrm{e}-04$ & 1.30 & $5.18 \mathrm{e}-05$ & 1.55 & $1.45 \mathrm{e}-05$ & 1.84 \\
1.02 & $8.36 \mathrm{e}-04$ & $4.10 \mathrm{e}-04$ & 1.03 & $1.80 \mathrm{e}-04$ & 1.19 & $6.89 \mathrm{e}-05$ & 1.39 & $2.16 \mathrm{e}-05$ & 1.67 \\
1.01 & $8.69 \mathrm{e}-04$ & $4.46 \mathrm{e}-04$ & 0.96 & $2.12 \mathrm{e}-04$ & 1.07 & $9.31 \mathrm{e}-05$ & 1.19 & $3.44 \mathrm{e}-05$ & 1.44 \\
1.00 & $9.00 \mathrm{e}-04$ & $4.83 \mathrm{e}-04$ & 0.90 & $2.49 \mathrm{e}-04$ & 0.95 & $1.27 \mathrm{e}-04$ & 0.97 & $6.38 \mathrm{e}-05$ & 0.99 \\
\hline 1.01 & $1.70 \mathrm{e}-03$ & $5.38 \mathrm{e}-04$ & 1.66 & $1.43 \mathrm{e}-04$ & 1.91 & $3.71 \mathrm{e}-05$ & 1.95 & $9.45 \mathrm{e}-06$ & 1.97 \\
1.00 & $1.70 \mathrm{e}-03$ & $5.38 \mathrm{e}-04$ & 1.66 & $1.43 \mathrm{e}-04$ & 1.91 & $3.71 \mathrm{e}-05$ & 1.95 & $9.45 \mathrm{e}-06$ & 1.97 \\
\hline
\end{tabular}

on the computational domain $x \in \Omega=(0,1)$. Here $\Gamma(x)$ is the classic Gamma function. We consider the initial condition

$$
u(x, 0)=x^{5},
$$

and the Dirichlet boundary conditions

$$
u(0, t)=0, \quad u(1, t)=\mathrm{e}^{-t},
$$

where $\beta \in[1,2]$. The exact solution is given by $\mathrm{e}^{-t} x^{5}$.

We measure the errors corresponding to the exact solution $u(x, t)$ in the discrete $L^{2}$ norm at $t=1$. Tables $2-4$ for $k=1,2,3$ demonstrate the errors and order of convergence both schemes. For the unstablized scheme, optimal convergence is observed for $\beta \in[1+\varepsilon, 2.0]$ with suboptimal convergence for $\beta=1.0$ as predicted by Theorem 4.2.

However, the results also clearly demonstrate that the optimal asymptotic order of convergence is recovered as $\beta \rightarrow 1$, for the stabilized scheme recovers the optimal order of convergence uniformly for $\beta \in[1,2]$.

We finally consider the question of how the spectral radius scales with $\beta$ and the spatial resolution. Illustrated in Table 5, we observe a fully discrete scaling for solving equation (1.1) as

$$
\Delta t \sim\left(\frac{h}{k^{2}}\right)^{\beta},
$$

where $\Delta t$ is the time step size and $k$ still is the order of the approximate polynomial. Provided the stabilization is taken as $L(h, \beta) \sim\left(k^{2} / h\right)^{\beta-1}$, this does not impact the scaling. This is in agreement with expectations based on the experience for integer values of $\beta$ [13]. 
TABLE 3. The error and order of convergence for second order polynomial approximation $(k=2)$. $M$ denotes the number of elements. For $\beta>1.1$ the results are independent of whether stabilization with $L(h, \beta)=h^{\beta}$ is included. Recovery of the optimal order of convergence is possible only with the stabilization as illustrated in the last two rows which show results with the stabilized scheme.

\begin{tabular}{|l|c|c|l|c|l|c|l|}
\hline \multirow{2}{*}{$\beta$} & $M=2^{3}$ & \multicolumn{2}{|c|}{$M=2^{4}$} & \multicolumn{2}{c|}{$M=2^{5}$} & \multicolumn{2}{c|}{$M=2^{6}$} \\
\cline { 2 - 8 } & error & error & order & error & order & error & order \\
\hline 2.00 & $4.92 \mathrm{e}-04$ & $6.62 \mathrm{e}-05$ & 2.89 & $8.61 \mathrm{e}-06$ & 2.94 & $1.09 \mathrm{e}-06$ & 2.98 \\
1.80 & $5.19 \mathrm{e}-04$ & $6.76 \mathrm{e}-05$ & 2.94 & $8.50 \mathrm{e}-06$ & 2.99 & $1.07 \mathrm{e}-06$ & 2.99 \\
1.50 & $5.53 \mathrm{e}-04$ & $7.27 \mathrm{e}-05$ & 2.93 & $9.08 \mathrm{e}-06$ & 3.00 & $1.12 \mathrm{e}-06$ & 3.02 \\
1.20 & $6.04 \mathrm{e}-04$ & $7.02 \mathrm{e}-05$ & 3.11 & $8.73 \mathrm{e}-06$ & 3.01 & $1.10 \mathrm{e}-06$ & 2.99 \\
1.10 & $3.04 \mathrm{e}-04$ & $8.28 \mathrm{e}-05$ & 1.88 & $9.80 \mathrm{e}-06$ & 3.08 & $9.74 \mathrm{e}-07$ & 3.33 \\
1.09 & $2.98 \mathrm{e}-04$ & $8.20 \mathrm{e}-05$ & 1.86 & $1.01 \mathrm{e}-05$ & 3.01 & $8.97 \mathrm{e}-07$ & 3.50 \\
1.08 & $2.92 \mathrm{e}-04$ & $8.10 \mathrm{e}-05$ & 1.85 & $1.05 \mathrm{e}-05$ & 2.95 & $7.99 \mathrm{e}-07$ & 3.71 \\
1.07 & $2.86 \mathrm{e}-04$ & $7.99 \mathrm{e}-05$ & 1.84 & $1.08 \mathrm{e}-05$ & 2.88 & $6.80 \mathrm{e}-07$ & 4.00 \\
1.06 & $2.80 \mathrm{e}-04$ & $7.87 \mathrm{e}-05$ & 1.83 & $1.12 \mathrm{e}-05$ & 2.82 & $5.51 \mathrm{e}-07$ & 4.34 \\
1.05 & $2.74 \mathrm{e}-04$ & $7.75 \mathrm{e}-05$ & 1.82 & $1.15 \mathrm{e}-05$ & 2.75 & $4.36 \mathrm{e}-07$ & 4.72 \\
1.04 & $2.69 \mathrm{e}-04$ & $7.62 \mathrm{e}-05$ & 1.82 & $1.18 \mathrm{e}-05$ & 2.69 & $3.63 \mathrm{e}-07$ & 5.02 \\
1.03 & $2.63 \mathrm{e}-04$ & $7.48 \mathrm{e}-05$ & 1.81 & $1.21 \mathrm{e}-05$ & 2.63 & $3.69 \mathrm{e}-07$ & 5.03 \\
1.02 & $2.57 \mathrm{e}-04$ & $7.34 \mathrm{e}-05$ & 1.81 & $1.23 \mathrm{e}-05$ & 2.58 & $4.28 \mathrm{e}-07$ & 4.85 \\
1.01 & $2.51 \mathrm{e}-04$ & $7.19 \mathrm{e}-05$ & 1.81 & $1.25 \mathrm{e}-05$ & 2.53 & $5.08 \mathrm{e}-07$ & 4.62 \\
1.00 & $2.46 \mathrm{e}-04$ & $7.04 \mathrm{e}-05$ & 1.81 & $1.26 \mathrm{e}-05$ & 2.48 & $5.79 \mathrm{e}-07$ & 4.45 \\
\hline 1.01 & $1.50 \mathrm{e}-05$ & $1.43 \mathrm{e}-06$ & 3.41 & $1.10 \mathrm{e}-07$ & 3.70 & $1.07 \mathrm{e}-08$ & 3.36 \\
1.00 & $1.52 \mathrm{e}-05$ & $1.43 \mathrm{e}-06$ & 3.39 & $1.10 \mathrm{e}-07$ & 3.71 & $1.06 \mathrm{e}-08$ & 3.36 \\
\hline
\end{tabular}

TABLE 4 . The error and order of convergence for third order polynomial approximation $(k=3)$. $M$ denotes the number of elements. For $\beta>1.1$ the results are independent of whether stabilization with $L(h, \beta)=h^{\beta}$ is included. Recovery of the optimal order of convergence is possible only with the stabilization as illustrated in the last two rows which show results with the stabilized scheme. For $M=14$, the convergence rate of the stabilized scheme exceeds 4 .

\begin{tabular}{|l|c|c|l|l|l|}
\hline \multirow{2}{*}{$\beta$} & $M=4$ & \multicolumn{2}{|c|}{$M=8$} & \multicolumn{2}{c|}{$M=12$} \\
\cline { 2 - 6 } & error & error & order & error & order \\
\hline 2.00 & $1.49 \mathrm{e}-04$ & $1.12 \mathrm{e}-05$ & 3.74 & $2.51 \mathrm{e}-06$ & 3.69 \\
1.80 & $1.56 \mathrm{e}-04$ & $1.13 \mathrm{e}-05$ & 3.79 & $2.48 \mathrm{e}-06$ & 3.73 \\
1.50 & $2.00 \mathrm{e}-04$ & $1.37 \mathrm{e}-05$ & 3.87 & $2.74 \mathrm{e}-06$ & 3.97 \\
1.20 & $3.89 \mathrm{e}-04$ & $2.98 \mathrm{e}-05$ & 3.71 & $5.16 \mathrm{e}-06$ & 4.33 \\
1.10 & $5.45 \mathrm{e}-04$ & $5.37 \mathrm{e}-05$ & 3.34 & $1.04 \mathrm{e}-05$ & 4.06 \\
1.09 & $5.65 \mathrm{e}-04$ & $5.78 \mathrm{e}-05$ & 3.29 & $1.14 \mathrm{e}-05$ & 4.00 \\
1.08 & $5.85 \mathrm{e}-04$ & $6.22 \mathrm{e}-05$ & 3.23 & $1.26 \mathrm{e}-05$ & 3.94 \\
1.07 & $6.05 \mathrm{e}-04$ & $6.72 \mathrm{e}-05$ & 3.17 & $1.40 \mathrm{e}-05$ & 3.89 \\
1.06 & $6.26 \mathrm{e}-04$ & $7.28 \mathrm{e}-05$ & 3.11 & $1.56 \mathrm{e}-05$ & 3.80 \\
1.05 & $6.48 \mathrm{e}-04$ & $7.89 \mathrm{e}-05$ & 3.04 & $1.74 \mathrm{e}-05$ & 3.72 \\
1.04 & $6.71 \mathrm{e}-04$ & $8.57 \mathrm{e}-05$ & 2.97 & $1.96 \mathrm{e}-05$ & 3.64 \\
1.03 & $6.94 \mathrm{e}-04$ & $9.32 \mathrm{e}-05$ & 2.90 & $2.22 \mathrm{e}-05$ & 3.54 \\
1.02 & $7.18 \mathrm{e}-04$ & $1.01 \mathrm{e}-04$ & 2.82 & $2.51 \mathrm{e}-05$ & 3.45 \\
1.01 & $7.43 \mathrm{e}-04$ & $1.11 \mathrm{e}-04$ & 2.75 & $2.86 \mathrm{e}-05$ & 3.34 \\
1.00 & $7.70 \mathrm{e}-04$ & $1.21 \mathrm{e}-04$ & 2.67 & $3.27 \mathrm{e}-05$ & 3.22 \\
\hline 1.01 & $3.25 \mathrm{e}-06$ & $1.38 \mathrm{e}-06$ & 3.84 & $6.79 \mathrm{e}-07$ & 3.89 \\
1.00 & $3.25 \mathrm{e}-06$ & $1.38 \mathrm{e}-06$ & 3.84 & $6.79 \mathrm{e}-07$ & 3.89 \\
\hline
\end{tabular}


TABLE 5. The time step sizes required with a fixed space step length $h=0.01$ for different values of $\beta$ first and second order polynomial approximations. Here 'S' denotes stable and ' $U$ ' unstable.

\begin{tabular}{lcccccc}
\hline$\Delta t$ (1st order) & $\Delta t$ (2nd order) & $\beta=1.0$ & $\beta=1.2$ & $\beta=1.5$ & $\beta=1.8$ & $\beta=2.0$ \\
\hline $2.0 d-2$ & $1.0 d-2$ & $\mathrm{~S}$ & $\mathrm{U}$ & $\mathrm{U}$ & $\mathrm{U}$ & $\mathrm{U}$ \\
$1.0 d-2$ & $5.0 d-3$ & $\mathrm{~S}$ & $\mathrm{~S}$ & $\mathrm{U}$ & $\mathrm{U}$ & $\mathrm{U}$ \\
$1.0 d-3$ & $1.0 d-3$ & $\mathrm{~S}$ & $\mathrm{~S}$ & $\mathrm{~S}$ & $\mathrm{U}$ & $\mathrm{U}$ \\
$5.0 d-4$ & $1.0 d-4$ & $\mathrm{~S}$ & $\mathrm{~S}$ & $\mathrm{~S}$ & $\mathrm{~S}$ & $\mathrm{U}$ \\
$1.0 d-4$ & $1.0 d-5$ & $\mathrm{~S}$ & $\mathrm{~S}$ & $\mathrm{~S}$ & $\mathrm{~S}$ & $\mathrm{~S}$ \\
\hline
\end{tabular}

We also note that in agreement with the theoretical analysis, both fluxes (3.15) and (3.16) work well for the two schemes for any $\beta \in[1,2]$. This may seem counterintuitive when $\beta=1$ since in this case one of the flux choices reflect down winding. This illustrates that when $\beta=1$ the single equation is equivalent to the system but in doing numerical computations a new mechanism (some kind of symmetry) is introduced when writing the pure diffusion equation as a system due to the introduction of the fluxes.

\section{CONCluding REMARKS}

We have proposed a new stabilized local discontinuous Galerkin method for the fractional diffusion equation, containing both limits of pure convection equation and pure diffusion. In the framework of fractional derivatives, the fractional diffusion equation 'bridges' the two classic equations and we have shown that a single scheme can be used to accurately solve both types of problems.

We have discussed two schemes in this paper, the second one being a modified version of the first one, obtained by adding a penalty term to the global term. Both schemes allow a theoretical and numerical optimal order of convergent when $\beta \in(1,2]$, but only a suboptimal order of convergence can be established rigorously in the extreme case $\beta=1$. For the stabilized scheme we can show numerically that optimal order of convergence for any $\beta \in[1,2]$ can be recovered. This is not, however, confirmed rigorously.

This paper is among the first to study and analyze the application of DG methods to spatial fractional derivatives, illustrating that DG methods have significant potential for problems with fractional derivatives due to their flexibility and ability to support high order accuracy while maintain a large degree of locality in the formulation. With the smoothness associated with solutions to fractional problems [10], the high order accuracy allows for a reduced number of degrees of freedom without impacting the accuracy. This is a substantial advantage due to the global nature of the fractional operators, suggesting a reduced computational cost over alternative methods, in particular for problems in higher dimensions. We hope to discuss this in more detail in future work.

Acknowledgements. We thank the reviewers for their helpful comments. The authors wish to express their gratitude to Drs B. Stamm and Y. Chen, both of Brown University, who provided valuable insight and suggestions during the early part of the work reported here.

\section{REFERENCES}

[1] R.A. Adams, Sobolev Spaces. Academic Press, New York (1975).

[2] E. Barkai, Fractional Fokker-Planck equation, solution, and application. Phys. Rev. E. 63 (2001) 046118.

[3] F. Bassi and S. Rebay, A high-order accurate discontinuous finite element method for the numerical solution of the compressible Navier-Stokes equations. J. Comput. Phys. 131 (1997) 267-279.

[4] P.L. Butzer and U. Westphal, An Introduction to Fractional Calculus. World Scientific, Singapore (2000).

[5] C.-M. Chen, F. Liu, I. Turner and V. Anh, A Fourier method for the fractional diffusion equation describing sub-diffusion. J. Comput. Phys. 227 (2007) 886-897. 
[6] B. Cockburn and C.-W. Shu, The local discontinuous Galerkin method for time-dependent convection diffusion systems. SIAM J. Numer. Anal. 35 (1998) 2440-2463.

[7] P. Castillo, B. Cockburn, D. Schötzau and C. Schwab, Optimal a priori error estimates for the hp-version of the local discontinuous Galerkin method for convection-diffusion problem. Math. Comput. 71 (2001) 455-478.

[8] P. Ciarlet, The Finite Element Method for Elliptic Problems. North-Holland, Amsterdam (1975).

[9] W.H. Deng, Numerical algorithm for the time fractional Fokker-Planck equation. J. Comput. Phys. 227 (2007) $1510-1522$.

[10] W.H. Deng, Finite element method for the space and time fractional Fokker-Planck equation. SIAM J. Numer. Anal. 47 (2008) 204-226.

[11] V.J. Ervin and J.P. Roop, Variational formulation for the stationary fractional advection dispersion equation. Numer. Methods Partial Differ. Eqs. 22 (2005) 558-576.

[12] J.S. Hesthaven and T. Warburton, High-order nodal discontinuous Galerkin methods for Maxwell eigenvalue problem. Roy. Soc. London Ser. A $\mathbf{3 6 2}$ (2004) 493-524.

[13] J.S. Hesthaven and T. Warburton, Nodal Discontinuous Galerkin Methods: Algorithms, Analysis, and Applications. SpringerVerlag, New York, USA (2008).

[14] X. Ji and H. Tang, High-order accurate Runge-Kutta (Local) discontinuous Galerkin methods for one- and two-dimensional fractional diffusion equations. Numer. Math. Theor. Meth. Appl. 5 (2012) 333-358.

[15] C.P. Li and W.H. Deng, Remarks on fractional derivatives. Appl. Math. Comput. 187 (2007) 777-784.

[16] X.J. Li and C.J. Xu, A space-time spectral method for the time fractional diffusion equation. SIAM J. Numer. Anal. 47 (2009) $2108-2131$.

[17] Y.M. Lin and C.J. Xu, Finite difference/spectral approximations for the time-fractional diffusion equation. J. Comput. Phys. 225 (2007) 1533-1552.

[18] W. McLean and K. Mustapha, Convergence analysis of a discontinuous Galerkin method for a sub-diffusion equation. Numer. Algorithms 52 (2009) 69-88.

[19] K. Mustapha and W. McLean, Discontinuous Galerkin method for an evolution equation with a memory term of positive type. Math. Comput. 78 (2009) 1975-1995.

[20] K. Mustapha and W. McLean, Piecewise-linear, discontinuous Galerkin method for a fractional diffusion equation. Numer. Algorithms 56 (2011) 159-184.

[21] K. Mustapha and W. McLean, Superconvergence of a discontinuous Galerkin method for the fractional diffusion and wave equation, arXiv:1206.2686v1 (2012).

[22] R. Metzler and J. Klafter, The random walk's guide to anomalous diffusion: A fractional dynamics approach. Phys. Rep. 339 (2000) $1-77$.

[23] C. Tadjeran and M.M. Meerschaert, A second-order accurate numerical method for the two-dimensional fractional diffusion equation. J. Comput. Phys. 220 (2007) 813-823.

[24] J. Yan and C.-W. Shu, A local discontinuous Galerkin method for KdV type equations. SIAM J. Numer. Anal. 40 (2002) 769-791. 